Research Article

\title{
Characterization and Efficacy of A Novel Formula of Insulin Chitosan Layered Duple Hydroxide-Nanohybrid for Oral Administration
}

\author{
Ahmed Abdel-Wahab El-Shahawy ${ }^{1}$, Adel Abdel-Moneim ${ }^{2}$, Zienab Esam Eldin ${ }^{3}$, \\ Ahmed Ismail Youssef², Zhraa Selem Gouda², Hanaa Ramadan², Eiman Amar², \\ Esraa Ashraf Taha ${ }^{2}$, Asmaa Mohamed Saied², Mohamed Zanaty ${ }^{4}$ \\ ${ }^{1}$ Department of Materials Science and Nanotechnology, Faculty of Postgraduate Studies for Advanced Sciences (PSAS), Beni-Suef \\ University, Egypt. \\ ${ }^{2}$ Division of Molecular Physiology, Faculty of Science, Beni-Suef University, Egypt. \\ ${ }^{3}$ Faculty of Veterinary Medicine, Beni-Suef University, Egypt. \\ ${ }^{4}$ Department of Biotechnology, Faculty of Postgraduate Studies for Advanced Sciences (PSAS), Beni-Suef University, Egypt. \\ Corresponding authors. E-mail: adel_men2020@yahoo.com; adel.hassan@science.bsu.edu.eg
}

Received: May 23, 2020; Accepted: Sep. I, 2020; Published: Oct. 23, 2020

Citation: Ahmed El-Shahawy, Adel Abdel-Moneim, Zienab Eldin, Ahmed Youssef, Zhraa Gouda, Hanaa Ramadan, Eiman Amar, Esraa Taha, Asmaa Saied, and Mohamed Zanaty. Characterization and Efficacy of A Novel Formula of Insulin Chitosan LDH-Nanohybrid for Oral Administration. Nano Biomed. Eng., 2020, 12(4): 297-305.

DOI: 10.5101/nbe.v12i4.p297-305.

\begin{abstract}
The development of an oral insulin therapy remains an ultimate goal to both enhance ease of use, and to provide therapeutic advantages rooted in its direct delivery to the portal vein and liver. The current study aimed to develop a novel formula for insulin oral administration against experimental diabetes in rats. Entrapped insulin (INS) between chitosan nanoparticles (CSNPs) and layered duple hydroxide (LDH) (LDH-INS-CSNPs) was chemically prepared, and characterized by X-ray diffraction, Fourier transformation infrared, high-resolution transmission electron microscope, field emission scanning electron microscope and Zeta potential measurements. The insulin release study was conducted in vitro, while the oral hypoglycemic effect of LDH-INS-CSNPs was investigated in vivo in diabetic rats. The prepared formulas revealed a variation in the spectra of characterization methods. The insulin entrapment between LDH and chitosan avoided the burst release of insulin and acid denaturation in the stomach and enzymatic degradation throughout the gut. Consequently, the blood glucose level of LDH-INS-CSNPs formula exhibited a marked hypoglycemic effect. The present work showed that the LDH-INS-CSNPs formula had a protective effect against enzymatic degradation, reduced insulin initial burst release, and enhanced bioavailability through the oral administration route. Interestingly, the presented formula could be an oral antidiabetic agent alternative to injectable insulin.
\end{abstract}

Keywords: Diabetes, Insulin, Oral administration, Bioavailability, Sustain release

\section{Introduction}

Diabetes Mellitus (DM) is a common metabolic condition. Repeated subcutaneous insulin injection is the standard treatment for controlling the blood glucose level in type 1 and type 2 diabetes mellitus patients [1]. However, current insulin subcutaneous injection causes local discomfort, patient noncompliance, hypoglycemia and hyperinsulinemia. For many physiological reasons, the oral bioavailability of insulin is low as it is a protein drug [2]. Previous studies showed that the strategy for effective oral 
insulin delivery is based on insulin protection against gastric and enzymatic degradation, allows insulin to be released into the large intestine and increases insulin absorption by opening the epithelial tight junction of the intestinal wall [3].

In the past few years, nano-carriers have opened a new landscape for the oral delivery of biomolecules [4]. The oral insulin administration has attracted the attention of large numbers of biomedical scientists. It could improve the loading performance of insulin on hydrophobic carriers by using soybean phospholipid [5], enteric coatings [6], enzyme inhibitors [7] and permeation enhancers [8] to enhance the absorption of insulin. Chitosan (CS), a cationic and biological polysaccharide, was designated a promising nanomaterial with extensive biomedical applications due to its biodegradable, biocompatible, mucoadhesive, nontoxic, and ability to target specific delivery properties $[9,10]$. Novel nano-materials extensively developed from CS have been progressively examined over the past decade, with potential applications for targeted drug delivery [11].

Recently, significant attention was given to the use of inorganic nanoparticles in drug delivery applications, since they have versatile attributes with wide availability, strong biocompatibility and rich surface functionality [12]. Besides, emerging bioinorganic hybrid systems, such as layered double hydroxides (LDH), are promising for drug delivery [13]. Also, it able to intercalate with different therapeutic compounds comprising conjugated linoleic acid [14], indole-3-acetic acid [15] and to show biocompatibility for several bio-applications [16].

Notably, medical researchers have recently committed to developing an oral delivery system of insulin for improved diabetes treatment [17], but until now, no successful outcome to avoid the mucosal barrier and increase the bioavailability, which are the main reasons for oral insulin failure [18]. Therefore, the present study aimed to prepare a new formula of insulin nano-hybrid carrier consisting of organic and inorganic constituents (LDH-INS-CSNPs), to achieve its sustained release and to increase its bioavailability via the oral administration route in diabetic rats.

\section{Experimental}

\section{Materials}

Sodium hydroxide $(\mathrm{NaOH})$, hydrochloric acid $(\mathrm{HCl})$, acetone, aluminum nitrate $\left(\mathrm{Al}\left(\mathrm{NO}_{3}\right)_{2} \cdot 9 \mathrm{H}_{2} \mathrm{O}\right)$, zinc nitrate $\left(\mathrm{Zn}\left(\mathrm{NO}_{3}\right)_{2} \cdot 6 \mathrm{H}_{2} \mathrm{O}\right)$, methanol, acetic acid, 1-Propanol, $\beta$-alanine, mono-potassium phosphate $\left(\mathrm{KH}_{2} \mathrm{PO}_{4}\right), \mathrm{NaH}_{2} \mathrm{PO}_{4} \cdot 12 \mathrm{H}_{2} \mathrm{O}$ and chitosan medium-MW (75 kDa) were purchased from LOBA Chemie, India. Streptozotocin (STZ) purchased from Sigma (SigmaAldrich Co., St. Louis, Missouri, USA).

\section{Preparation of Zn-AI LDH}

Zn-Al LDH was prepared as mentioned previously [19]. Briefly, $\mathrm{Zn}\left(\mathrm{NO}_{3}\right)_{2} \cdot 6 \mathrm{H}_{2} \mathrm{O}$ and $\mathrm{Al}\left(\mathrm{NO}_{3}\right)_{3} \cdot 9 \mathrm{H}_{2} \mathrm{O}$ with a molar ratio $4: 1$ were dissolved in $100 \mathrm{~mL}$ distilled water, and $\mathrm{NaOH}$ (1 M) was added by dropwise until complete precipitation at $\mathrm{pH}$ (8-9). The precipitate suspension lasted under vigorous stirring at $60{ }^{\circ} \mathrm{C}$ for $24 \mathrm{~h}$. Finally, it was filtered, washed until pH 7 , and dried at $40^{\circ} \mathrm{C}$ for $24 \mathrm{~h}$.

\section{Preparation of Zn-AI LDH-chitosan}

As mentioned in the previous investigation [20], the $\mathrm{Zn}$-Al LDH-chitosan composite was formed via the co-precipitation method; a mix of $\mathrm{Al}\left(\mathrm{NO}_{3}\right)_{3} \cdot 9 \mathrm{H}_{2} \mathrm{O}$ and $\mathrm{Zn}\left(\mathrm{NO}_{3}\right)_{2} \cdot 6 \mathrm{H}_{2} \mathrm{O}(\mathrm{Al} / \mathrm{Zn}=1 / 3$ molar ratio) was dissolved in a solution of distilled water and 1-propanol. Then $35 \mathrm{~mL}$ from this mixture were inserted into a blend of $25 \mathrm{~mL}$ distilled water, $10 \mathrm{~mL}$ 1-propanol and $30 \mathrm{~mL}$ of chitosan $(0.25 \mathrm{~g} / \mathrm{l})$ and $\beta$-alanine $(0.005 \mathrm{M})$ with a high magnetic stirring; $\mathrm{NaOH}$ solution $(0.5 \mathrm{M})$ was added dropwise to adjust $\mathrm{pH}$ 7.5. The mixture was transferred into a Teflon-lined autoclave and heated at $120{ }^{\circ} \mathrm{C}$ for $18 \mathrm{~h}$. The precipitate was filtered, washed many times to remove inorganic ions, and dried in vacuum at $60^{\circ} \mathrm{C}$ for $24 \mathrm{~h}$.

\section{Preparation of insulin-loaded chitosan nanoparticles INS-CSNPS}

Chitosan nanoparticles were prepared as described in previous publications [21, 22]. Then, $8 \mathrm{mg}$ of chitosan nanoparticles were dissolved in $4 \mathrm{~mL}$ of an aqueous solution of acetic acid ( $1 \% \mathrm{v} / \mathrm{v})$ and the $\mathrm{pH}$ value of the resulting solution was 3.4. Insulin (2 mg) was dissolved in $0.01 \mathrm{M} \mathrm{NaOH}(0.5 \mathrm{~mL})$ and mixed with $0.9 \%(\mathrm{w} / \mathrm{V})$ typically sodium tripolyphosphate (TPP) aqueous solution $(1 \mathrm{~mL})$. Then the mixture was slowly added dropwise to the chitosan solution under stirring.

\section{Preparation of novel LDH-INS-CSNPS}

Firstly, insulin was loaded on LDH according to the method of Zhao et al. [23]. A chitosan solution that comprised $8 \mathrm{mg}$ of chitosan, $4 \mathrm{~mL}$ of an aqueous solution of acetic acid (1\% v/v) and $\mathrm{NaOH}$ at $\mathrm{pH} 6.5$ was prepared. Simultaneously, a solution of $\mathrm{Al} \mathrm{Zn}$ 
LDH-insulin (2 mg) plus $0.5 \mathrm{~mL}$ of $0.01 \mathrm{M} \mathrm{NaOH}$ was added to the chitosan solution by a drop wise; the total mixture was stirred in a magnetic stirrer for about 1 hour at room temperature. Then, poloxamer 188 (0.1\% $\mathrm{w} / \mathrm{v}$ ), and $1 \mathrm{~mL}$ of $0.9 \%$ (w/w) TPP solution were added dropwise to the mixture under stirring for 10 minutes. In the final, the system was centrifuged with the addition of mannitol $(0.05 \% \mathrm{w} / \mathrm{v})$ and the sediment was then freeze-dried.

\section{Characterization of the prepared materials}

$\mathrm{X}$-ray diffraction (XRD) technique characterized the crystallinity of the mentioned formulas using $\mathrm{Cu} \mathrm{K} \alpha$ radiation $(\lambda=1.54 \AA)$ in a $2 \theta$ scan range $5-80^{\circ}$. Fourier transformation infrared (FTIR) spectra were recorded on Bruker Vertex 70 FTIR spectrometer in the range from 400 to $4000 \mathrm{~cm}^{-1}$. High-resolution transmission electron microscope (HRTEM) images were acquired using (JEM-2100; JEOL, Japan), with an acceleration voltage of $200 \mathrm{kV}$. Field emission scanning electron microscope images (FESEM; JSM 6400 device, IEQL) were obtained. The particle sizes and zeta potentials were measured using a ZS90 Zetasizer instrument (Malvern, UK).

\section{Insulin entrapment efficiency measurement}

$5 \mathrm{~mL}$ of LDH-INS-CSNPs formula were centrifuged, and the supernatant was diluted to 50 $\mathrm{mL}$; the HPLC method was implied to estimate the insulin level in the supernatant. The chromatographic conditions followed the methodology of Cuilet al. [24]. Briefly, HPLC (SDV 505, Anyang, South Korea) equipment was employed to determine the amount of free insulin, the used detector $210 \mathrm{~nm}$, C8 column (Chrompack, Edinburg, United Kingdom 4.6 mm $\times 15$ $\mathrm{cm}, 5 \mu \mathrm{m}$ ), and mixture aqueous solution of $\mathrm{NaH}_{2} \mathrm{PO}_{4}$, $\mathrm{Na}_{2} \mathrm{SO}_{4}$, and acetonitrile $\mathrm{C}_{2} \mathrm{H}_{3} \mathrm{~N}$ in a ratio of 35:35: 30 constituted the mobile phase. The flow rate of the mobile phase was $1 \mathrm{~mL} / \mathrm{min}$, and the volume of the sample was $20 \mu \mathrm{L}$. The final $\mathrm{pH}$ was adjusted to 3 with $\mathrm{H}_{3} \mathrm{PO}_{4}$, the detector wavelength was $214 \mathrm{~nm}$, and the temperature of the oven was $35^{\circ} \mathrm{C}$. The concentration of insulin was calculated from a standard curve, and the measured insulin was the free fraction of the total insulin added to the system. The entrapment efficiency of insulin (IE) was calculated from equation $\mathrm{IE}=\left(\mathrm{I}_{\mathrm{t}}-\mathrm{I}_{\mathrm{f}}\right) /$ $I_{t}$, where $I_{t}$ is the total insulin, and If the free insulin in the supernatant.

\section{Insulin release profile from LDH-INS-CSNPs formula}

$5 \mathrm{mg}$ of LDH-INS-CSNPs formula were joined to $5 \mathrm{~mL}$ of phosphate buffer solution ( $\mathrm{pH} \mathrm{7.4)} \mathrm{and}$ stirred in a shaker $50 \mathrm{rpm}$ at $37{ }^{\circ} \mathrm{C}$. Samples were taken at $0,1,2,4,6$, and $8 \mathrm{~h}$ and centrifuged. Insulin was detected by HPLC in the supernatant, and the accumulated concentration of the released insulin from the mentioned formula was estimated.

\section{Hypoglycemic effect in vivo study}

Adult male Wistar rats (120-140 g) were used in the present study supplied by the Holding Company for Biological Products and Vaccines (VACSERA, Cairo, Egypt). All animal experiments were approved by the Institutional Animal Care and Use Committee (IACUC), Beni-Suef University, Egypt. STZ 50 mg/kg b. wt. was dissolved in cold citrate buffer at pH 4.5 [25]. Diabetes mellitus was experimentally induced in rats fasted for 16 h by intraperitoneal injection dissolved STZ. One week post-STZ-injection, animals of fasting glucose concentration $\geq 150 \mathrm{mg} / \mathrm{dL}$ were included in the experiment.

\section{Experimental design}

Rats were divided into 8 groups $(\mathrm{N}=8)$ as follows: Normal control, Diabetic rats, Diabetic rats+ INS oral, Diabetic rats+ INS subcutaneous injection (sc), Diabetic rats + CSNPs, Diabetic rats+ INS-CSNPs, Diabetic rats+ LDH-CSNPs, and Diabetic rats+ LDHINS-CSNPs. The dose was equivalent to (20 IU/kg b.wt. insulin). All doses were given daily via gastric intubation for 2 weeks, except for the INS-sc group. Glucose level was estimated in the blood sample, which collected from the lateral tail vein of each rat after the second week of treatment using a glucometer (CERA-CHECK ${ }^{\mathrm{TM}}$ 1070).

\section{Statistical analysis}

The Statistical Package for the Social Sciences (IBM SPSS for WINDOWS 7, version 22; SPSS Inc., Chicago) was used for the statistical analysis. All statistical comparisons were made by one- way analysis of variance (ANOVA) followed by Duncan's method for post-hoc analysis. The obtained data were expressed as mean \pm standard error (SE). Values with $P$ $<0.05$ were considered statistically significant.

\section{Results and Discussion}

\section{Structure and characterization}

Insulin therapy had significant advances in purity and the capability to give further physiologic regulation of blood glucose levels. New delivery approaches have been proposed to overcome barriers and to attain better 
oral bioavailability, including the use of surfactants, permeation enhancers, enteric coatings, carrier systems, and chemical modifications of insulin. The presented study designed oral administration of LDHINS-CSNPs formula as an alternative to the current injectable free insulin. Chitosan was chosen to enhance insulin absorption in the intestinal tract, while LDH was selected to attain the sustained release of insulin.

Fig. 1 displayed the X-ray diffraction (XRD) patterns of the formed materials and loaded insulin. The peak position (angle of diffraction) illustrates the crystal-structure while the peak height is a resolution of the crystallinity. The XRD spectrum of Zn-Al LDH matched with ICDD card No. (00-058-0178), and illustrated characteristics diffraction peaks at $2 \theta$ $=9.90^{\circ}$ and $19.874^{\circ}$ corresponded to the diffraction planes (003), and (006) planes. The (006) peak marked the hydrotalcite-type and well-ordered crystalline materials, while the (003) peak indicated the basal of an interlayer anion in LDH materials [26]. The observed peaks of LDH were closely matched with the results of Balcomb et al. [27]. The XRD of chitosan exhibited the semi-crystalline nature of chitosan with two distinct peaks at $2 \theta=10^{\circ}$ and $50^{\circ}$ due to (020) and (110), respectively. The XRD of LDH-INS-CSNPs showed a diffraction peak shifting of (003) and (006) specifying the synergy between chitosan and LDH [28]. Besides, the disappearance of the small diffracted peaks of LDH may refer to one of the following reasons: removal of water molecules, rearrangement of $\mathrm{Zn} / \mathrm{Al}$ ions, and / or adsorption of insulin molecules on the surface of LDH. Insulin's peaks are thought to have a much lower intensity compared with the LDH and chitosan phases and could not be observed in the XRD diffractogram. The crystallinity of LDH-INS-CSNPs was somewhat distorted as shown in the selected area electron diffraction image, Fig.3(b). These alterations observed implied the interaction between the three entities.

Fig. 2 showed the FTIR spectra of the prepared materials and loaded insulin at different wavenumbers. $\mathrm{Zn}-\mathrm{Al}$ LDH displayed a wide band at $3444 \mathrm{~cm}^{-1}$ that referred to $v(\mathrm{OH})$ group stretching vibration in the brucite-related sheets and the interlamellar water

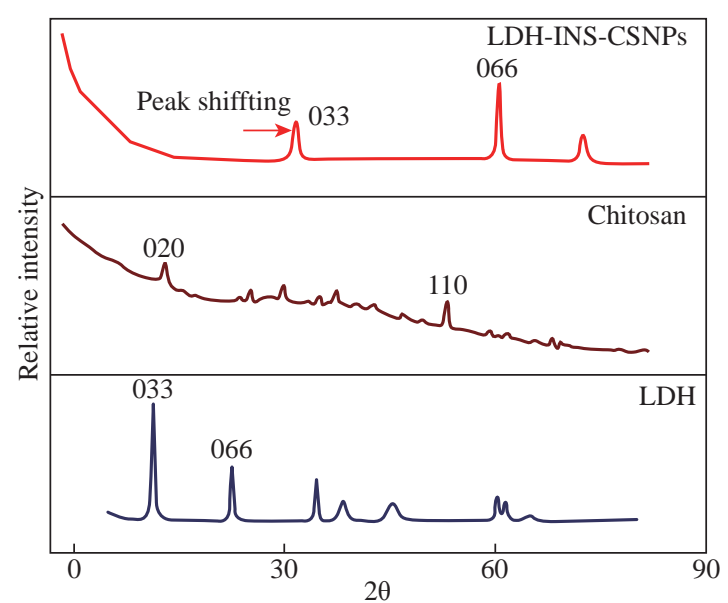

Fig. 1 XRD pattern of the prepared materials shows the microstructure and the crystallinity of $\mathrm{Zn}$-Al layered double hydroxide ( $\mathrm{LDH})$, chitosan nanoparticles, and entrapped insulin between chitosan and $\mathrm{LDH}$, revealing variations in the diffraction peaks positions and width.

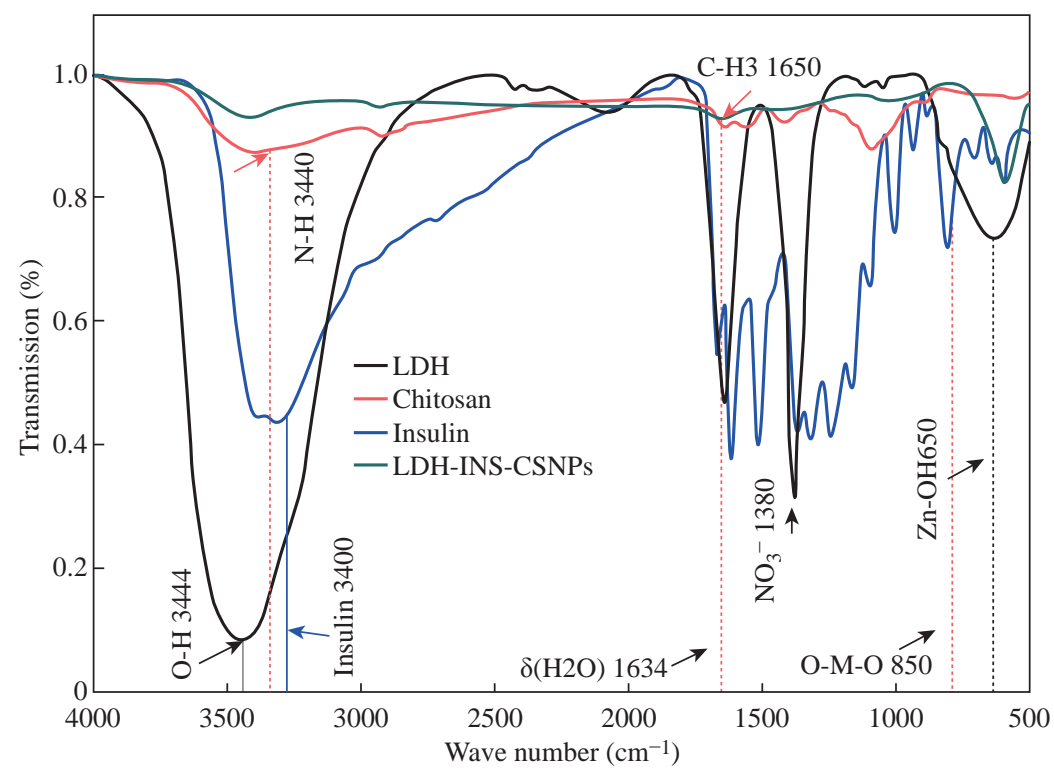

Fig. 2 FTIR spectra of the Zn-Al layered double hydroxide (LDH), chitosan nanoparticles, insulin, and entrapped insulin between chitosan and LDH, the characteristic peaks of insulin at $3400-3100 \mathrm{~cm}^{-1}$ still present with low intensities confirming the presence of insulin in the Ins-CSNPs-LDH formula. 
molecules. The band's broadening was attributed to the formation of hydrogen bonds [29]. The appeared band at $1380 \mathrm{~cm}^{-1}$ was assigned to the $v 3$ (antisymmetric) stretching vibration of the $\mathrm{NO}^{3-}$ groups in the $\mathrm{LDH}$ interlayer. The feeble band at $1634 \mathrm{~cm}^{-1}$ could be assigned to the $\delta\left(\mathrm{H}_{2} \mathrm{O}\right)$ bending vibration of the interlayer water. The band approximately at $850 \mathrm{~cm}^{-1}$ arose from $\mathrm{O}-\mathrm{M}-\mathrm{O}$ vibrations in the brucite-like layers $(\mathrm{M}=\mathrm{Al})$, where, the weak band at $650 \mathrm{~cm}^{-1}$ was induced by the lattice vibration modes corresponding to the translation vibration of $\mathrm{Zn}-\mathrm{OH}$ [30]. The pure chitosan spectrum showed basic characteristic peaks of chitosan, the band at $3440 \mathrm{~cm}^{-1}$ was related to $\mathrm{NH}$ stretching vibration, peak at $1650 \mathrm{~cm}^{-1}$ was assigned to $\mathrm{CH}_{3}$ symmetrical in the amide group, a band at 1600 $\mathrm{cm}^{-1}$ was attributed to the bending vibration of $\mathrm{N}-\mathrm{H}$ group, the band at $1741 \mathrm{~cm}^{-1}$ of $\mathrm{C}=\mathrm{O}$, and the peak at $1629 \mathrm{~cm}^{-1}$ was due to amide (I) [31]. The band at 2880 $\mathrm{cm}^{-1}$ (C-H stretch), the weak band at $1654 \mathrm{~cm}^{-1}\left(\mathrm{NH}_{2}\right.$ deformation), and the band at $1153 \mathrm{~cm}^{-1}$ was assigned to (bridge-O-stretch) [32]. The FTIR spectrum of the insulin revealed a weak band at $1654 \mathrm{~cm}^{-1}$ and a strong band at $3400-3100 \mathrm{~cm}^{-1}$ that are characteristic of insulin [33]. These bands are still present with low intensities in the spectrum of LDH-INS-CSNPs confirming the presence of insulin in the formula.

It is well-known that the oral administration of insulin is the main challenge because insulin's stability is weak due to its easily degraded in the gastrointestinal tract by the action of acids, alkalis, and proteases. Besides, it is a large molecule and poorly absorbed through the intestinal membrane. Consequently, the bioavailability of insulin is low. Practical solutions for this issue and some researchers have reported. Sharma et al. [34] showed that loading insulin on nanospheres protected insulin from degradation, and enhanced its uptake via Peyer's patches in the mucosa of the small intestine. Moreover, Iyer et al. [35] reported that oral administration of insulin-loaded nanospheres was more effective than intraperitoneal injection. Notably, Schipper et al. [36] stated that chitosan is a good absorption enhancer for poorly absorbable drugs, and boosted the insulin absorption across the intestinal mucosa. However, the fundamental limitation of that research was the burst release of the insulin from the chitosan in the acidic medium of the stomach. A potential interpretation for this shortcoming is the stomach $\mathrm{pH}$ solution, $\mathrm{pH} 3.2<\mathrm{pKa} 6.5$ of chitosan, and gives the chitosan a positive charge by protonation of the amino group. Likewise, under this condition, insulin is positively charged due to the stomach $\mathrm{pH}$ value is lower than the pKa value (5.3) of insulin [37]. Theoretically, the same charges would create a repulsive force leading to the burst release of insulin. The limitations of some previous studies encouraged us to conduct our preliminary work to investigate the LDH-INS-CSNPs formula as one of the suggested solutions to the problem of oral administration and the burst release of insulin.

In the current study, the LDH-INS-CSNPs formula was prepared at $\mathrm{pH} 6.5$, the value was greater than the pKa value of the insulin and smaller than that of chitosan, so that the chitosan was positively charged, while insulin was negatively charged, while the LDH surface was positively charged. Theoretically, we can say that the insulin entrapped between the LDH from one side and chitosan from the other side, and the calculated insulin entrapment efficiency was $80.5 \pm 3.1 \%$. The electrostatic interaction between the particles due to the negatively charged insulin and the positively charged chitosan and LDH, boosted the stability of the formula, which confirmed by the value of the zeta potential of LDH-INS-CSNPs 38.1 $\pm 0.5 \mathrm{mV}$. Furthermore, the electrostatic interaction maintained the entity structure, which appeared in the HRTEM image (Fig. 3(a)), However, the magnitude of the measured zeta potential of LDH-INS-CSNPs

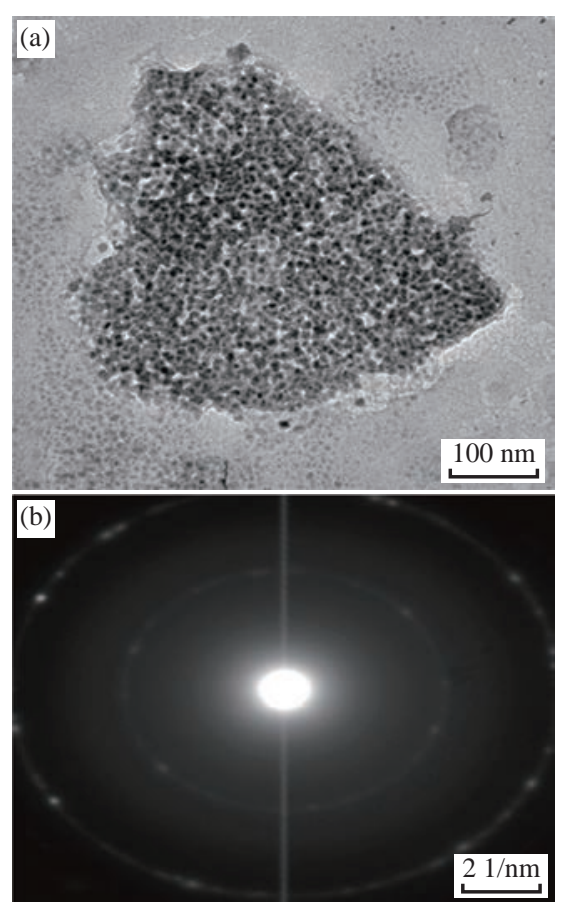

Fig. 3 (a) HRTEM image of LDH-INS-CSNPs formula showing aggregation state between the particles; (b) Selected area electron diffraction (SAED) revealing low crystallinity of the prepared formula. 
was not sufficient to achieve repelling, resulting in an aggregation state among the particles and an increase in the size, as shown in the 3 dimensions FESEM image of the LDH-INS-CSNPs formula, Fig. 4. Consequently, the Zeta Sizer and size distribution were not suitable for dynamic light scattering measurements.

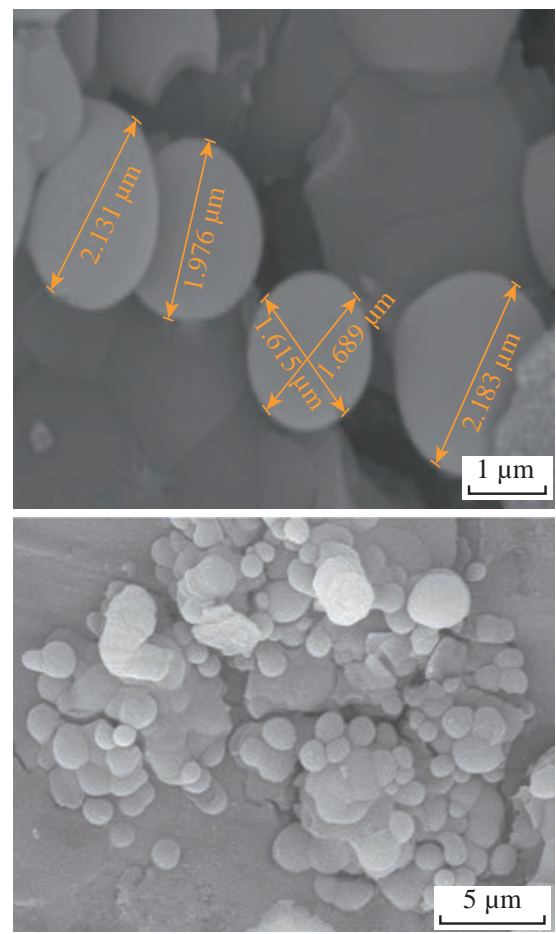

Fig. 4 SEM images showing measurements and accumulation of LDH-INS-CSNPs formula.

It is worth noticing that the chitosan with mediumMW (75 kDa) was employed in the presented research based on previous studies [38,39], which indicated that low and molecular weight of chitosan enhanced the solubility, loading, and release of hydrophobic drugs. Besides, chitosan has been extensively investigated for its potential absorption enhancer across the intestinal epithelium. The mucoadhesive property of chitosan is mediated by its ability to spread over the mucous layer and additionally through its positive ionic interactions with the negative charges of the mucus or the cell surface membranes. On the other hand, LDHs have attractive anion-exchangeability, strong adsorption of negative charges, owing to its inherent positive charge, large surface-to-volume ratio and biocompatibility, and able to carry anionic biomolecules.

Fig. 5 presents the cumulative percentage release of free insulin and the entrapped insulin from the LDHINS-CSNPs formula versus time. After one hour from the start of the release experiment, the cumulative percentage of the released insulin from the LDHINS-CSNPs formula was about $5 \%$, while that of free

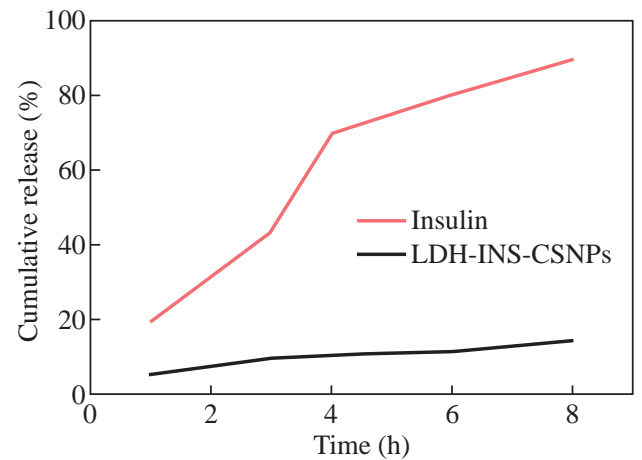

Fig. 5 In vitro liberation profile of insulin entrapped between chitosan nanoparticles and $\mathrm{Zn}$-Al layered double hydroxide (LDH) revealing sustained release for $8 \mathrm{~h}$.

insulin recorded about 20.0\%. After 2 h, the percent release of the entrapped insulin was $18.0 \%$, and the free insulin was $37.24 \pm 4.5 \%$. The cumulative percentage release of the entrapped insulin increased slowly up to $51.8 \pm 6.9$ after $8 \mathrm{~h}$ compared to $89.8 \pm 5.8 \%$ of the free insulin. The slow release of the entrapped insulin was due to that the low solubility of chitosan at $\mathrm{pH} 7.4$, and decreased the release rate in the first $2 \mathrm{~h}$, avoiding the burst release. Another explanation is that the insulin took a long time to separate from the surface of LDH and the linkage with chitosan before diffusing into the solution. The separation of insulin from the surface of LDH might be through ion exchange reaction between $\mathrm{LDH}$ with chloride or phosphate ions present in the biological fluids. However, a drawback of LDHs materials was the poor kinetics control of the drug release. For solving this issue, alterations in the synthesis methods and surface modification with polymers or surfactants have been reported [40]. Interestingly, the release of insulin at mentioned $\mathrm{pH}$ that mimics intestinal medium, implied that the prepared formula was stable and protected the insulin from enzymatic and acidic degradation in the stomach.

\section{Effect of LDH-INS-CSNPs on blood glucose concentration}

Regarding the in vivo study in the current study, Fig. 6 illustrates the statistical analysis of glucose fasting and $2 \mathrm{~h}$ postprandial concentrations in the examined groups after two weeks of treatments. The figure revealed that fasting blood glucose concentration of diabetic rats significantly increased compared to normal control, while all treated groups showed nonsignificant changes as compared to diabetic one. Concerning postprandial measurements, the INS-oral, CSNPs, and LDH-CSNPs treated groups revealed a non-significant change in relative to diabetic one, while both of the INS-sc and LDH-INS-CSNPs were 


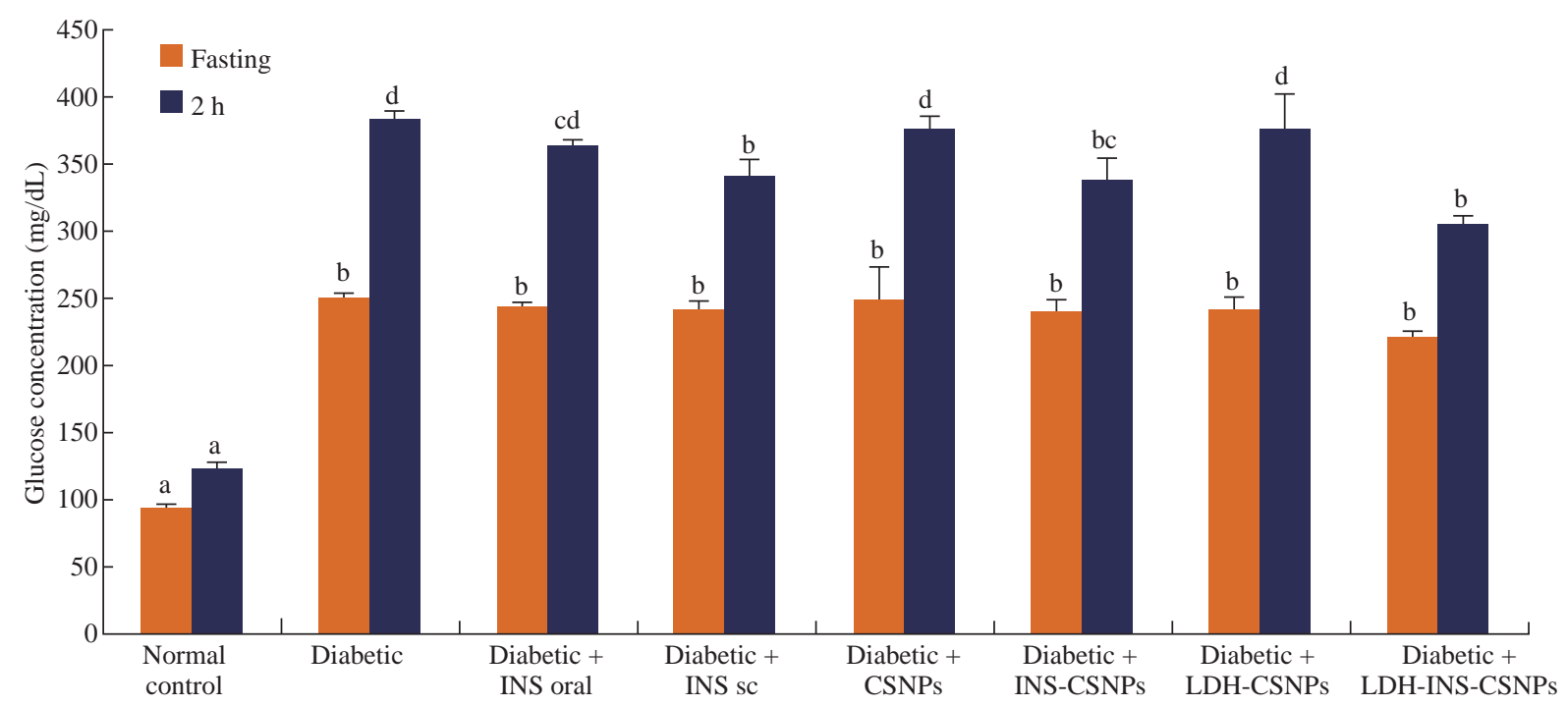

Fig. 6 In vivo effect of insulin treatment via subcutaneous and oral route treatments on blood glucose levels in diabetic rats. Means which not share the same superscript symbol(s) are significantly different at $\mathrm{P}<0.05$.

the most significant hypoglycemic groups. One of the suggested explanations for this result was that the presented formula LDH-INS-CSNPs started to dissolve into the intestine alkaline medium and the positive charge of chitosan induced better adhesion with the negatively charged lining the intestinal mucosa and facilitated the insulin absorption as well as fostered the bioavailability of insulin; hence, the efficiency of oral LDH-INS-CSNPs formula was higher than free oral insulin (INS-oral) as shown in Fig. 6. The current data were in parallel with Bruno et al. [41], who investigated the possibility of reducing enzymatic and absorption barriers to protein and peptide drugs. Also, Liu et al. [42] evaluated the potential of different protease inhibitors on the intestinal absorption and degradation of insulin.

Importantly, the issue of oral insulin formula is not fully optimized and is still a challenge. New strategies have been developed to enhance oral insulin delivery; however, many questions are still pending, and this is an important issue for future research. Regarding the study limitation, the future work will target the toxicity investigation and in vivo pharmacokinetics studies and optimization of the LDH-INS-CSNPs formula with the addition of suitable enzyme inhibitors and absorption enhancers, as well as exploring the different hypoglycemic pathways.

\section{Conclusions}

Although oral insulin nanoparticles were significantly improved, clinical challenges still exist.
The oral insulin nanoparticles are needed to enhance the bioavailability and sustain release. In this context, the current study presented LDH-INS-CSNPs as a new formula to improve the insulin entrapment efficiency, avoided the alimentary canal degradation, reduced the burst release, and enhanced bioavailability. The oral administration revealed a significant hypoglycemic effect compared to free oral and injectable insulin. This study suggested that the LDH-INS-CSNPs formula might be considered as a suitable alternative to the current injectable insulin.

\section{Acknowledgments}

The authors are grateful to all members of the Department of Materials Science and Nanotechnology, Faculty of Advanced Sciences Postgraduate Studies, and all members of the Department of Zoology, Faculty of Science, University of Beni-Suef, Egypt, for helping the study. The authors are grateful to the Academy of Scientific Research and Technology (ASRT), Egypt, for providing the research grant.

\section{Funding}

This study was supported by the Academy of Scientific Research and Technology (ASRT) under Grant for Graduation Projects.

\section{Conflict of interests}

The authors declare that there is no conflict of interest. 


\section{References}

[1] O. Korsgren, B. Nilsson, Improving islet transplantation: A road map for a widespread application for the cure of persons with type I diabetes. Curr Opin Organ Tran, 2009, 14: 683-687.

[2] Y.Y. Luo, X.Y. Xiong, Y. Tian, et al., A review of biodegradable polymeric systems for oral insulin delivery. Drug Deliv, 2016, 23(6): 1882-1891.

[3] K. Al Rubeaan, M. Rafiullah, and S. Jayavanth, Oral insulin delivery systems using chitosan-based formulation: a review. Expert Opin Drug Deliv, 2016, 13(2): 223-237.

[4] S. Gupta, A. Jain, M. Chakraborty, et al., Oral delivery of therapeutic proteins and peptides: a review on recent developments. Drug Deliv, 2013, 20: 237-246.

[5] P. Li, H.M. Nielsen, and M. Fano, A. Müllertz, Preparation and characterization of insulin-surfactant complexes for loading into lipid-based drug delivery systems. J Pharm Sci., 2013, 102: 2689-2698.

[6] X. Zhao, C. Shan, Y. Zu, et al., Preparation, characterization, and evaluation in vivo of Ins-SiO(2)HP55 (insulin-loaded silica coating HP55) for oral delivery of insulin. Int J Pharm, 2013, 454: 278-284.

[7] M. Werle, B. Loretz, D. Entstrasser, et al., Design and evaluation of a chitosan-aprotinin conjugate for the peroral delivery of therapeutic peptides and proteins susceptible to enzymatic degradation, J Drug Target, 2007, 15: 327-333.

[8] Y. Zhang, W. Wei, P. Lv, et al., Preparation and evaluation of alginate-chitosan microspheres for oral delivery of insulin. Eur J Pharm Biopharm, 2011, 77: 11-19.

[9] J. Li, C. Cai, J. Li, et al., Chitosan-Based Nanomaterials for Drug Delivery. Molecules, 2018, 23(10): 2661. doi:10.3390/molecules23102661

[10] C. Saikia, P. Gogoi, and T.K. Maji, Chitosan: A Promising Biopolymer in Drug Delivery Applications. J Mol Genet Med, 2015, S4: 006. doi:10.4172/1747-0862.S4-006.

[11] J. Lin, Y. Li, Y. Li, et al., Drug/Dye-loaded, multifunctional PEG-chitosan-iron oxide nanocomposites for methotraxate synergistically self-targeted cancer therapy and dual model imaging. ACS Appl Mater Interfaces, 2015, 7: 11908-11920.

[12] A. Ravindran-Girija, and S. Balasubramanian., Theragnostic potentials of core/shell mesoporous silica nanostructures. Nanotheranostics, 2019,3(1): 1-40.

[13] Li Y, Liu D, Ai H, et al., Biological evaluation of layered double hydroxides as efficient drug vehicles. Nanotechnology, 2010, 21(10): 105101.

[14] K. Zhang, Z.P. Xu, J. Lu, et al., Potential for layered double hydroxides-based, innovative drug delivery systems. Int J Mol Sci, 2014, 15(5): 7409-7428.

[15] L.P.F. Benício, R.A. Silva, J.A. Lopes, et al., Layered double hydroxides: Nanomaterials for applications in agriculture. Rev Bras Ciênc Solo, 2015, 39: 1-13.

[16] D. Hwan, P. Goeun, and C. Ho-Choy., Bio-layered double hydroxides nanohybrids for theranostics applications. Photofunctional layered materials (structure and bonding), $2015^{\text {th }}$ edition, Springer, 2015: 137-175.

[17] E.B. Souto, S.B. Souto, J.R. Campos, et al., Nanoparticle delivery systems in the treatment of diabetes complications. Molecules., 2019, 20;24(23):4209.

[18] Y. Yang, H. Zhu, J. Wang, et al., Enzymatically disulfide crosslinked chitosan/hyaluronic acid layer-by-layer selfassembledmicrocapsules for redox-responsive controlled release of protein. ACS Appl Mater Interfaces, 2018, 10: 33493-33506.

[19] N. Tajuddin, J. Manayil, M. Isaacs, et al., Alkali-free ZnAl layered double hydroxide catalysts for triglyceride transesterification. Catalysts, 2018, 8 (12): 667.
[20] P.R. Wei, S.H. Cheng, W.N. Liao, et al., Synthesis of chitosan-coated near-infrared layered double hydroxide nanoparticles for in vivo optical imaging. J Mater Chem, 2012, 22: 5503-5513.

[21] N.K. Al-Nemrawi, S.M. Alsharif, K.H. Alzoubi, et al., Preparation and characterization of insulin chitosannanoparticles loaded in buccal films. Pharm Dev Technol, 2019, 24(8): 967-974.

[22] K. Divya1, and M.S. Jisha., Chitosan nanoparticles preparation and applications. Environ Chem Lett, 2018, 16: 101-112.

[23] P. Zhao, Y. Liu, L. Xiao, H. Deng, Y. Du, and X. Shi, Electrochemical deposition to construct nature inspired multilayer chitosan/layered double hydroxides hybrid gel for stimuli responsive release of protein. J Mater Chem B, 2015, 3(38): 7577-7584.

[24] F. Cui1, L. Zhang, J. Zheng, et al., A study of insulin-chitosan complex nanoparticles used for oral administration. J Drug Del Sci Tsch, 2004, 14(6): 435439.

[25] F.G. Soufi, D. Mohammad-Nejad, and H. Ahmadieh, Resveratrol improves diabetic retinopathy possibly through oxidative stress-nuclear factor $\kappa \mathrm{B}$-apoptosis pathway. Pharmacol Rep, 2012, 64(6): 1505-1514.

[26] A.R. Sotiles, N.A.G. Gomez, S.C. Silva, et al., Layered Double Hydroxides with the Composition Mn/Al-SO4-A (A=Li, Na, K; Mn:Al ca.1:1) as Cation Exchangers. $J$ Braz Chem Soc, 2019, 30(9):1807-1813.

[27] B. Balcomb, M. Singh, and S. Singh., Synthesis and characterization of layered double hydroxides and their potential as nonviral gene delivery vehicles. Open Chem J,2015, 4(2): 137-145.

[28] D. Sokol, DEL Vieira, A. Zarkov, et al., Sonication accelerated formation of Mg-Al-phosphate layered double hydroxide via sol-gel prepared mixed metal oxides. Scientific Reports, 2019, 9: 10419. https://doi. org/10.1038/s41598-019-46910-5.

[29] R.C. Zeng, X.T. Li, Z.G. Liu, et al., Corrosion resistance of Zn-Al layered double hydroxide/poly(lactic acid) composite coating on magnesium alloy AZ31. Front Mater Sci, 2015, 9(4): 355-365.

[30] T. Qi, B.J. Reddy, H. He, et al., Synthesis and infrared spectroscopic characterization of selected layered double hydroxides containing divalent $\mathrm{Ni}$ and Co. Mater Chem Phys, 2008, 112(3): 869-875.

[31] S. Babakhani, Z.A. Talib, M.Z. Hussein, et al., Optical and thermal properties of Zn/Al-layered double hydroxide nanocomposite intercalated with sodium dodecyl sulfate. J Spectrosc, 2014, 2014: 467064.

[32] N.A. Mohamed, and N.A. Abd El-Ghany., Synthesis and antimicrobial activity of some novel terephthaloyl thiourea cross-linked carboxymethyl chitosan hydrogels. Cellulose, 2012, 19: 1879-1891.

[33] L. Nielsen, S. Frokjaer, J.F. Carpenter, et al., Studies of the structure of insulin fibrils by Fourier transform infrared (FTIR) spectroscopy and electron microscopy. $J$ Pharm Sci, 2001, 90(1): 29-37.

[34] G. Sharma, A.R. Sharma, J.S. Nam, et al., Nanoparticle based insulin delivery system: the next generation efficient therapy for Type 1 diabetes. J Nanobiotechnology, 2015,13: 74. doi:10.1186/s12951-015-0136-y.

[35] H. Iyer, A. Khedkar, and M. Verma., Oral insulin- a review of current status. Diabetes Obes Metab, 2010, 12: 179-185.

[36] N.G. Schipper, K.M. Vârum, P. Stenberg, et al., Chitosans as absorption enhancers of poorly absorbable drugs. 3 : Influence of mucus on absorption enhancement. Eur $J$ Pharm Sci, 1999, 8(4): 335-343.

[37] N. Saïed, and M. Aïder., Zeta Potential and Turbidimetry Analyzes for the evaluation of chitosan/phytic acid 
complex formation. J Food Res, 2014, 3(2): 71-81.

[38] Y. Shao, L. Li, X. Gu, et al., Evaluation of chitosananionic polymers based tablets for extended-release of highly water-soluble drugs, Asian J Pharm Sci, 2015, 10(1): 24-30.

[39] M.A. Mohammed, J.T.M. Syeda, K.M. Wasan, et al. An overview of chitosan nanoparticles and its application in non-parenteral drug delivery, Pharmaceutics, 2017, 9: 53.

[40] J.O.Y. Mathew, S.J. Iyengar, J. Chakraborty, and S. Ghosh. Layered double hydroxide using hydrothermal treatment: morphology evolution, intercalation and release kinetics of diclofenac sodium. Front Mater Sci. 2017, 11(4): 395-409.

[41] B.J. Bruno, G.D. Miller, and C.S. Lim., Basics and recent advances in peptide and protein drug delivery. Ther Deliv,
2013, 4(11): 1443-1467.

[42] H. Liu, W.S. Pan, R. Du, et al., Effects of protease inhibitors on intestinal absorption of insulin. Yaо Хие Xиe Bao, 2004, 39(2): 140-143.

Copyright $\left({ }^{\complement}\right.$ Ahmed El-Shahawy, Adel Abdel-Moneim, Zienab Eldin, Ahmed Youssef, Zhraa Gouda, Hanaa Ramadan, Eiman Amar, Esraa Taha, Asmaa Saied, and Mohamed Zanaty. This is an open-access article distributed under the terms of the Creative Commons Attribution License, which permits unrestricted use, distribution, and reproduction in any medium, provided the original author and source are credited. 